\title{
Altered temporal stability in dynamic neural networks underlies connectivity changes in neurodevelopment
}

Matthew J. Brookes ${ }^{1 *}$, Madeleine J. Groom², Lucrezia Liuzzi ${ }^{1}$, Ryan M. Hill ${ }^{1}$, Helen J.F. Smith ${ }^{2}$, Paul M. Briley ${ }^{2}$, Emma L. Hall ${ }^{1}$, Benjamin A.E. Hunt ${ }^{3}$, Lauren E. Gascoyne ${ }^{1}$, Margot J. Taylor ${ }^{3}$, Peter F. Liddle $^{2}$, Peter G. Morris ${ }^{1}$, Mark W. Woolrich ${ }^{4+}$ and Elizabeth B. Liddle ${ }^{2+}$

${ }^{1}$ Sir Peter Mansfield Imaging Centre, School of Physics and Astronomy, University of Nottingham, University Park, Nottingham, NG7 2RD, United Kingdom.

${ }^{2}$ Centre for Translational Neuroimaging in Mental Health, Institute of Mental Health, School of Medicine, University of Nottingham, Jubilee Campus, Triumph Road, Nottingham, UK.

${ }^{3}$ Diagnostic Imaging, Hospital for Sick Children, 555 University Avenue, Toronto, Ontario, Canada M5G $1 \times 8$.

4 Oxford Centre for Human Brain Activity, Wellcome Centre for Integrative Neuroimaging, Department of Psychiatry, University of Oxford.

+ Indicates authors that contributed equally to this paper

\section{"Correspondence to:}

Dr M. J. Brookes,

Sir Peter Mansfield Imaging Centre,

School of Physics and Astronomy,

University of Nottingham,

University Park,

Nottingham NG7 2RD

E-mail: matthew.brookes@nottingham.ac.uk

Pages: 28

Words: $\quad 12,109$

Figures: 5

Tables: $\quad 4$

Running Title: Functional connectivity, network dynamics, and neurodevelopment 


\section{ABSTRACT:}

Network connectivity is an integral feature of human brain function, and characterising its maturational trajectory is a critical step towards understanding healthy and atypical neurodevelopment. Here, we used magnetoencephalography (MEG) to investigate both stationary (i.e. time averaged) and rapidly modulating (dynamic) electrophysiological connectivity, in participants aged from mid-childhood to early adulthood (youngest participant 9 years old; oldest participant 25 years old). Stationary functional connectivity (measured via inter-regional coordination of neural oscillations) increased with age in the alpha and beta frequency bands, particularly in bilateral parietal and temporo-parietal connections. Our dynamic analysis (also applied to alpha/beta oscillations) revealed the spatiotemporal signatures of 8 dynamic networks; these modulate on a $100 \mathrm{~ms}$ time scale, and temporal stability in attentional networks was found to increase with age. Significant overlap was found between age-modulated dynamic networks and inter-regional oscillatory coordination, implying that altered network dynamics underlie age related changes in functional connectivity. Our results provide novel insights into brain network electrophysiology, and lay a foundation for future work in childhood disorders.

\section{HIGHLIGHTS:}

We studied static and dynamic connectivity change between mid-childhood and adulthood

Static (time averaged) connectivity increases with age in alpha and beta bands

Connectivity changes with age are strongest in attentional networks

Temporal stability in attentional networks was found to increase with age.

Changing network dynamics underlies changes in time averaged functional connectivity 


\section{INTRODUCTION:}

Recent years have seen a shift in our approach to brain mapping, from the study of discrete functional regions to the identification of networks in which multiple regions work in concert. The networks and underlying connectivities that have emerged from such studies are now recognised as being of fundamental importance to our understanding of healthy and atypical brain function. However, most studies focus on adults, missing pivotal changes in structural and functional architecture that occur during childhood and adolescence. Such investigation has the potential to reveal how brain networks mature, and to place this in the context of changing brain structure, behaviour and cognitive ability. Moreover, characterisation of network development is vital if we are to understand disorders, such as autism, that begin in childhood and are thought to involve altered functional connectivity (Leung et al. 2014; Supekar 2013; Ye et al. 2014; You 2013). Here we use magnetoencephalography (MEG) to track the development of electrophysiological connectivity from mid childhood to early adulthood.

The extant evidence for network changes in neurodevelopment comes largely from functional magnetic resonance imaging (fMRI) and suggests that networks typically observed in adults change significantly with development (Grayson and Fair 2017). For example, in a study of 5-8 year old children, networks supporting cognitive processing were found to be fragmented compared to adults (de Bie et al. 2012). Conversely, networks supporting sensorimotor and visual processing were found to exist in a relatively mature state. This is consistent with behavioural evidence of differential rates of development across different areas of cognitive function (Huizinga, Dolan, and van der Molen 2006). Other findings (Marek et al. 2015) suggest that between network integration also changes throughout development. Such studies have led the way in beginning to characterise the developing connectome. However, in fMRI, functional connectivity is defined by relationships between blood oxygenation level dependent (BOLD) signals from different regions. The BOLD signal is an indirect measure of neural activity, based upon haemodynamic changes; it has poor time resolution and cannot assess the electrophysiological mechanisms that mediate connectivity. It follows that a more complete understanding of the developmental trajectory of networks requires electrophysiological measurements.

MEG measures magnetic fields induced outside the head by current flow in the brain. Mathematical modelling of these fields enables construction of images showing changes in neural current over time. Recent years have seen the development of methods that capture connectivity using MEG data and these have allowed independent discovery of networks that, in many cases, match spatially those emerging from fMRI (e.g. (Brookes, Woolrich, et al. 2011; Fries 2015; Hall et al. 2014; Hipp et al. 2012; de Pasquale et al. 2010)). Such studies, alongside animal literature, show that coordination of neural oscillations (rhythmic electrical activity synchronised across cell assemblies) in well characterised frequency bands is a likely mechanism by which connectivity is mediated (Engel et al. 2013; Fries 2015). This opens up an exciting opportunity to investigate how synchrony between oscillations across a wide spectral range supports a hierarchy of electrophysiological networks. Interestingly, neural 
oscillations change significantly throughout development. For example, low frequency (delta/theta) resting state oscillatory amplitude is higher in younger participants (Clarke et al. 2001; Gasser et al. 1988). Task induced changes in higher frequency oscillations also differ; e.g. the post-movement beta modulation is diminished in young children (Gaetz et al. 2010). The effect of age on oscillatory mediated connectivity was shown, for the first time, in a recent study by Schafer et al (Schäfer et al. 2014). Via assessment of connectivity between networks identified a priori using fMRI, the authors showed significant increases in coordination of alpha and beta band oscillations within and across regions. However, elucidation of the changes in structural morphology of the connectome, and the relationship between connectivity and oscillatory amplitudes remains largely unexplored.

The major advantage of MEG (over $\mathrm{fMRI}$ ) is the ability to capture rapid (sub-second) network dynamics. The majority of connectivity studies are based upon an assumption of stationarity; i.e. connectivity between regions is characterised by a single parameter derived over many minutes (or even hours) of data. However, increasing evidence (Allen et al. 2014; Brookes et al. 2014; de Pasquale et al. 2010; de Pasquale et al. 2015; Hutchison et al. 2013; O'Neill, Barratt, et al. 2015; O'Neill, Bauer, et al. 2015; Vidaurre et al. 2016) suggests that the connectivities underlying brain networks change with time; this agrees with the notion that the brain, as a dynamical system, continually forms and dissolves networks on a timescale commensurate with cognition. Whilst the latency and longevity of the BOLD response obfuscates fast network dynamics, MEG has no such limitation and has been used to demonstrate connectivity changes on a timescale of seconds (O'Neill et al. 2017). Moreover, a paper by Baker et al. (Baker et al. 2014) used a Hidden Markov Model (HMM) to identify points in time at which unique patterns of electrical activity occur. In this way, brain 'states' were revealed, typically lasting $\sim 100 \mathrm{ms,}$ which correspond spatially to functional networks observed in fMRI. This was the first demonstration that networks modulate on a millisecond timescale. Further, slower time-scale functional connectivity was shown to be underpinned by these states with, for example, high functional connectivity in the sensorimotor network driven by increased occupancy of the corresponding state. How these rapid dynamics change throughout development remains an open and important question.

In this paper, we acquire resting state MEG data in a cross-sectional sample of healthy participants between the ages of 9 and 25 years (i.e. from mid-childhood to early-adulthood). We parcellate the brain in a manner that provides coverage of the cortex, and assess stationary (time averaged) functional connectivity between regions. In an independent analysis we use a HMM to assess network dynamics across the same parcellation. We test three hypotheses: i) Global (whole brain) stationary connectivity will increase significantly with age, in a frequency specific manner. ii) These connectivity changes will be spatially inhomogeneous; specifically, given the age range studied (in which primary sensory function is established) connectivity increases will occur in brain networks primarily associated with cognition and attention. iii) Altered network dynamics will underlie changes in functional connectivity: Specifically, the spatial signature of changing stationary connectivity will agree with one or more dynamic networks, whose temporal properties will change with brain maturation. 


\section{METHODS:}

\section{Data Acquisition:}

51 healthy volunteers (22 male, 29 female) took part in the study, which was approved by the University of Nottingham Faculty of Medicine \& Health Sciences Ethics Committee. Volunteers aged 16 and over provided written informed consent. Parental consent was obtained for volunteers under 16, who provided verbal assent. All participants underwent a structural MRI scan and multiple sessions of MEG acquisition. All participants were also tested for IQ. Note that in study design we aimed for 50 subjects to give $90 \%$ statistical power to detect a medium size correlation ( $\alpha=0.05$; two tailed test; $\mathrm{G}^{*}$ power). We aimed to minimise any systematic variation of $I Q$ with age at recruitment. We also aimed to distribute participants approximately equally across the 9-25 year age range (see Supplementary Information (SI) Table S3).

\section{MRI acquisition}

The anatomical MRI was acquired using a Philips Achieva 3T MRI system running an MPRAGE sequence ( $1 \mathrm{~mm}$ isotropic MPRAGE TE/TI/TR=8/960/3000ms). The result was a $\mathrm{T}_{1}$ weighted structural image which was used for subsequent source localisation of MEG data.

\section{MEG acquisition}

MEG data were recorded using the third order synthetic gradiometer configuration of a 275 channel CTF MEG system, at a sampling rate of $600 \mathrm{~Hz}$ and with a $150 \mathrm{~Hz}$ low pass anti-aliasing filter. The MEG system is housed inside a magnetically shielded room and all data were recorded with the participant supine. To reduce movement and increase comfort, a combination of head padding to the front, back and sides of the head was used with the aim of centralising the head within the sensor array. An additional custom made glass-fibre insert for the MEG helmet was also available for use with participants with smaller heads. This reduced the internal dimensions of the MEG helmet, thus making it easier to position a participant's head approximately equidistant from all MEG sensors. This is important as it ensures approximately equal signal to noise ratio (SNR) for all brain regions. Critically, in studies of this type, confounds are possible due to smaller heads in younger subjects. Specifically, smaller heads mean the brain is further from the sensors, causing a drop in signal to noise ratio and potentially a systematic error. Here, the effect of head size on signal strength across our cohort was assessed, and this was shown to not pose a significant problem (See SI Figure S1). MEG data were recorded whilst participants were at rest, and undertaking a working memory task. However, in this paper, only the resting state data are reported.

In the resting state recording, volunteers were asked to lie awake but at rest, with their eyes open for 5 minutes, and 'think of nothing'. To avoid eye movement, participants were asked to fixate on a marker which was placed at the centre of a back projection screen positioned approximately $35 \mathrm{~cm}$ in front of the participant. Prior to acquisition, three head position indicator (HPI) coils were placed at the nasion 
and pre-auricular points. These coils were energized continuously throughout MEG data acquisition to allow ongoing assessment of the head location. Prior to MEG, a 3D digitiser system (Polhemus, Colchester, Vermont, USA) was used to generate a 3D head shape for each subject, which included the locations of the HPI coils. Surface matching of the digitised head shape to a head shape extracted from the participants' MR images then allowed complete coregistration between brain anatomy and MEG sensor geometry.

\section{MEG Data Analysis:}

MEG data were initially inspected visually. A single participant (male, aged 19 years) was removed from the study due to artefacts throughout the recording. In the remaining 50 participants, the 300 s of data were divided into 30 'trials', each 10s in duration. Trials deemed to contain excessive interference, for example generated by electrical activity in eyes or muscles were removed. Parameters from the continuous motion tracking were analysed and a tolerance of $7 \mathrm{~mm}$ applied; of the 50 participants, 4 exhibited motion in excess of this threshold and trials were removed in which motion exceeded $7 \mathrm{~mm}$ from the mean head position. Following these analyses, a maximum of 3 trials had been removed from any one participant; this data removal was mirrored across all participants to ensure equivalent data duration (and hence reliability of connectivity measures) for all participants (Liuzzi et al. 2016). No relationship between age and movement was found.

\section{Brain Parcellation and source localisation}

To calculate a cortex-wide representation of functional connectivity, the cortex was parcellated into 78 regions according to the AAL atlas (Tzourio-Mazoyer et al. 2002). Following this, a beamformer (Robinson and Vrba 1998) was employed to generate a single time-course representing electrophysiological activity at the centre of mass of each region. A scalar linearly constrained minimum variance beamformer was used with covariance computed within a $1 \mathrm{~Hz}-150 \mathrm{~Hz}$ frequency window and a time window spanning the whole experiment, to minimise covariance matrix error (Brookes et al. 2008). Regularisation was applied to the covariance matrix using the Tikhonov method with a regularisation parameter equal to $5 \%$ of the maximum eigenvalue of the unregularised covariance matrix. The forward model was based on a dipole approximation (Sarvas 1987) and a multiple local sphere head model (Huang 1999). Dipole orientation was determined using a non-linear search for optimum SNR. Normalisation was applied (Hall et al. 2013) to eliminate the effect of the increase of noise with depth into the head. For any single participant, this results in a single timecourse of electrophysiological activity per brain region, hence a $78 \times f \Delta$ matrix whose rows contain the regional timecourses. ( $f$ represents sampling frequency and $\Delta(=270 s)$ is the experimental duration following removal of noisy trials). These regional signals were frequency filtered into 4 bands; theta $(4-8 \mathrm{~Hz})$; alpha $(8-13 \mathrm{~Hz})$ beta $(13-35 \mathrm{~Hz})$ and gamma $(35-70 \mathrm{~Hz})$ and used for the subsequent functional connectivity computations. Note that use of the beamformer in this way also acts to effectively reduce artifacts (e.g. the cardiac artifact) in the data (Brookes, Hale, et al. 2011; Sekihara et al. 2004). 


\section{Stationary Functional connectivity calculation}

Our aim was to infer stationary functional connectivity between all possible pairs of the $78 \mathrm{AAL}$ regions within each frequency band. However, a potential confound is that beamformer-derived timecourses from two regions may exhibit correlation purely due to 'signal leakage'. Leakage is a collective term which takes into account spatial blurring (e.g. due to the ill-posed inverse problem) and mislocalisation of sources (e.g. due to an inaccurate forward solution). It usually manifests as falsely inflated connectivity estimates between regions. Although this is a significant problem, leakage has been well studied and a number of methods for its reduction have been devised (Brookes, Woolrich, and Barnes 2012; Hipp et al. 2012; Nolte et al. 2004; Stam, Nolte, and Daffertshofer 2007; Wens et al. 2015). Most methods rely on the fact that leakage manifests as a zero-time lag linear summation of underlying signals and, for this reason, orthogonalisation of beamformer projected signals results in a marked reduction of leakage, albeit at the cost of true zero-phase-lag connectivity. Here, we employed a multivariate symmetric orthogonalisation (Colclough et al. 2015) involving two steps: First, a set of orthonormal time-courses that are closest to the data, and for which there is a simple analytic solution, is found. Second, the solution is finessed by iteratively adjusting the lengths and orientations of the orthogonal vectors until the solution is as close as possible to the uncorrected timecourses. The result is a $78 \times \mathrm{f} \Delta$ data matrix whose rows contain the orthogonalised time series for all $78 \mathrm{AAL}$ regions. It is important to note that this methodology does not correct for inaccuracies due to the ill posed MEG inverse problem; i.e. it cannot reconstruct sources that have already been blurred together in source reconstruction. Nevertheless this method has been shown to significantly ameliorate the leakage problem. For a more in depth discussion of limitations see (Brookes, Woolrich, and Barnes 2012; Colclough et al. 2015; Hipp et al. 2012).

Following leakage reduction, the orthogonalised band limited oscillations within each regional signal were Hilbert transformed, to derive an analytic signal. The absolute value of the analytic signal was then computed to yield the envelope of instantaneous oscillatory amplitude as a function of time in each frequency band of interest (theta, alpha, beta and gamma). This is henceforth known as the Hilbert envelope.

To reduce artefacts due to muscle activity, the regional timecourses were filtered into the $120 \mathrm{~Hz}-150 \mathrm{~Hz}$ band. (This was higher than any neural activity of interest but would capture accurately the magnetomyogram artefacts). The Hilbert envelope of these magnetomyogram data was then computed and regressed from the Hilbert envelopes of data in the frequency bands of interest, to reduce the effects of muscle artefact on connectivity (see Appendix 2). The corrected Hilbert envelope data for each frequency band (i.e. theta, alpha beta and gamma) were low pass filtered at $1 \mathrm{~Hz}$. This is because previous work indicates that envelope connectivity is strongest at these low frequencies (Brookes, Hale, et al. 2011; Brookes, Woolrich, et al. 2011; Hall et al. 2014; Hipp et al. 2012; O'Neill, Barratt, et al. 2015). 
Finally, connectivity was estimated as the Pearson correlation coefficient between all possible regional pairs of low-pass filtered oscillatory envelopes. This procedure generated four $78 \times 78$ weighted adjacency matrices per participant; these matrices represent the inter-regional connectivity between each of the 78 regions and every other region, for each of the four frequency bands.

Whole-brain relations between oscillatory amplitude, connectivity strength, and age A procedure was introduced to test for significant changes in neural oscillatory amplitude and functional connectivity with age, across the whole cortex.

- To examine global oscillatory amplitude, for each frequency band and participant, the mean amplitude of oscillations in each band (i.e. the mean Hilbert amplitude) was computed. These values were averaged across regions, resulting in a single value representing the oscillatory amplitude, for each frequency band and participant.

- To examine global connectivity, for each band, weighted adjacency matrices were summed in both dimensions to capture a single measure representative of the overall strength of whole brain connectivity, for each frequency band.

These computations resulted in 4 amplitude values and 4 connectivity strength values per participant. These values were plotted as a function of age across participants and relationships between amplitude/connectivity and age tested via a model fit. (Note that, in this particular case, the elements of our connectivity matrices were positive - and hence a simple summation of elements yields total connectivity strength. In cases where matrix elements are negative their absolute value should be taken before summation.)

We hypothesised an increase in functional connectivity with age. Previous work employed a linear model to test for such changes (Schäfer et al. 2014), however a body of literature also suggests that curvilinear relationships may be expected (Grimm, Ram, and Hamagami 2011). For this reason, we tested two separate models to characterize age related increases: a linear fit in which the rate of change with age is constant, and a non-linear monotonic fit in which the rate of change varies but the direction does not. Specifically, if $y$ represents our data for all 50 participants (either connectivity or amplitude, in some frequency band) and $x$ denotes age, then our linear model was given by $y=a_{1} x+a_{2}$,

and our non-linear monotonic model was given by

$y=-b_{1} / x+b_{2}$

In order to select the most appropriate model, we first tested for a significant fit to the data (see below). In cases where both fits were significant, the coefficient of determination $\left(R^{2}\right)$ was computed and the model for which this value was highest was considered the best. Note that both models have the same number of free parameters meaning that there is no confound of degrees of freedom. In cases in which 
neither of these models fitted the data, a third quadratic fit, which can capture a peak or trough in connectivity or amplitude with age, was also tested. Our quadratic model was given by $y=c_{1} x^{2}+c_{2} x+c_{3}$.

Even though the models represented by Equations 2 and 3 are non-linear, both can be written as linear functions of their respective parameters (i.e. for the purpose of parameter estimation they can be expressed as linear since e.g. in Equation 2, $y$ is a linear function of $1 / x$ ). This means each model could be formulated as a design matrix and model fits implemented using a general linear model (GLM). Note that the parameters $a_{1}, b_{1}$ and $c_{1,2}$ denote the absolute strength of the relationship between age and oscillatory amplitude or connectivity strength.

To assess the statistical significance of the best-fitting model in each case, a permutation approach was employed. We reasoned that if age had no effect on the measured (amplitude or connectivity) data then ages could be assigned randomly to each participant, and this would have no effect on the estimated parameters $a_{1}, b_{1}$ and $c_{1,2}$. We therefore constructed a series of plots showing oscillatory amplitude or connectivity versus "pseudo-age", where pseudo-age was defined simply by shuffling randomly the real ages across participants. On each iteration we repeated the GLM fitting procedure, and re-estimated the parameter fits. In this way, an empirical null distribution was derived for the parameter(s) of interest representing the values that would be obtained if age was meaningless. The parameter fits derived from the real data were then compared to the null distribution to derive an empirical p-value. 100,000 iterations of this procedure were implemented and a two tailed test was employed. In all cases, statistical significance was assigned as $p<0.05$, corrected for multiple comparisons across the four frequency bands, and across model fits using the Bonferroni approach. For the linear and non-linear-monotonic fits, we required that the parameters $a_{1}$ and $b_{1}$ fell in the tails of the null distribution. For the quadratic fit, two separate p-values were derived representing the squared and linear terms, i.e. the statistical significance of $c_{1}$ and $c_{2}$ respectively. For significance, we required the quadratic term to be statistically significant. To assess the effect of any statistical outliers in the data, this procedure was applied with and without outlier removal (where outliers were defined as points falling outside a range defined by the mean value (of amplitude or connectivity) plus or minus twice the standard deviation over subjects.

\section{The spatial morphology of connectivity change with age}

In the analyses above, connectivity was collapsed across the whole brain. However correlation between envelopes was measured between all possible pairs of the $\left(n_{a a l}=\right) 78 \mathrm{AAL}$ regions, resulting in a total of $\left(\left(n_{\text {aal }}^{2}-n_{\text {aal }}\right) / 2=\right) 3003$ possible connections - thus allowing derivation of the spatial signature of changing connectomics. To this end, for each connection, we plotted connectivity versus age, across participants, and employed either a linear, non-linear-monotonic or quadratic model (depending on which was significant at the whole brain level) to assess the strength of the ageconnectivity relationship. In this way, we obtained fitted parameters (either $a_{1}, b_{1}$ or $c_{1,2}$ ), representing 
the magnitude of the age-connectivity change, for every connection. These fits were used to form a matrix, termed the age-connectivity matrix, representing the spatial signature of the maturation of functional connectivity. In the case of linear or non-linear-monotonic relationships, elements of the ageconnectivity matrix were formed from a single fitted parameter (either $a_{1}$ or $b_{1}$ ), facilitating a direct assessment of the absolute change in connectivity with age. For a quadratic relationship, there are two parameters of interest, $c_{1}$ and $c_{2}$ representing squared and linear relationships with age, respectively. Here, rather than mapping each parameter independently we computed the standard deviation of the model, termed $s t d_{\text {quad. }}$ Note that this represents a quantitative assessment of connectivity change with age and is linearly related to $c_{1}$ and $c_{2}$. Importantly, mapping $a_{1}, b_{1}$ or $s t d_{\text {quad }}$ gives a matrix which is representative of the absolute change in connectivity with age, but says nothing of the quality of the model fit to the data. For this reason, for all 3003 connections, we applied our permutation test (as described above); connections where the age-connectivity relationship was not significant (i.e. p>0.05) were excluded from visualization (See also SI Figure S2).

\section{Network dynamics and neurodevelopment: the Hidden Markov Model}

Exploiting the excellent temporal resolution of MEG, we aimed to test whether age related changes in rapid network dynamics were related to the derived changes in stationary connectivity. To this end, we employed a Hidden Markov Model (Baker et al. 2014; Rezek and Roberts 2005; Woolrich et al. 2013) which is known to facilitate access to dynamic networks that fluctuate on a time scale of $\sim 100 \mathrm{~ms}$. As with the stationary connectivity analysis, data were parcellated according to the same 78 AAL regions and a single electrophysiological timecourse for each region derived using the same beamformer approach. Symmetric orthogonalisation (Colclough et al. 2015) was used to reduce any effect of signal leakage between regions. Data were frequency filtered in the $8-30 \mathrm{~Hz}$ band; this was for two reasons: Firstly, since previous work (Baker et al. 2014) using this same HMM employed a broad frequency range (integrating across traditional frequency bands (alpha, beta etc.)) we reasoned that the same broad band approach should be taken here. Secondly, our stationary analysis showed consistent effects of age in both the alpha $8-13 \mathrm{~Hz}$ and beta $13-30 \mathrm{~Hz}$ bands (see below), thus combining those bands was appropriate. Envelope data were derived using the Hilbert envelope approach and temporally down-sampled to $f_{\text {down }}=30 \mathrm{~Hz}$ (to increase computational efficiency). Data were then concatenated across subjects yielding a single $78 \times N_{\text {subs }} f_{\text {down }} \Delta$ matrix containing all of the envelope data for all subjects and regions. This matrix was used for the HMM inference.

For the HMM itself, we assume that brain activity is characterised by a relatively small number of "states" and that, at any single point in time, the brain exists in one of these states. Note that states are mutually exclusive. We further assume that the states themselves cannot be imaged directly (i.e. they are hidden) but that they can be inferred based upon measured observables (in this case, the concatenated parcellated envelope data). In addition, we assume that the underlying sequence of states is Markovian; i.e. the brain's current state depends only on its previous state, rather than a 
complete history of past states. Each state was described by a multivariate normal distribution with a $(78 \times 1)$ mean vector and a $(78 \times 78)$ covariance matrix. We used variational Bayes (VB) inference on the HMM (Rezek and Roberts 2005) to derive the full posterior distribution on the model parameters (i.e. we obtained a probabilistic description of the likelihood of the unobserved state parameters, and state transition probabilities, conditional on the measured data). In addition to this we determined, for every time-point, which of the derived states the brain was most likely in. This was done using Viterbi decoding (Baker et al. 2014; Woolrich et al. 2013). The result is a binary timecourse for each state, showing whether, for any one point in time, that state was the most likely. A HMM with 8 states was inferred (Baker et al. 2014). Code for the HMM inference is available from [https://github.com/OHBAanalysis/HMM-MAR].

The resulting 8 binary state timecourses were decomposed to obtain summary statistics. Specifically, for each state and each subject we estimated:

i) Fractional occupancy: The fraction of the total recording time that the brain was in each state.

ii) State lifetime: The mean time spent in each state on a single visit.

iii) State interval: The mean time between state visits

In addition, state maps were defined. For each brain region, we computed the partial correlation of the state timecourse with the envelope signal for that region (Baker et al. 2014). High positive correlation designates brain regions whose envelopes increase when the state is active. Conversely, high negative correlation designates brain regions whose envelope decreases when the state is active. State maps were made for both the whole subject cohort (partial correlation on the concatenated data) and also for each subject individually (partial correlation using data separated across subjects). In the latter case, partial correlation values were summed across regions to derive global measures representing the overall change in amplitude envelope related to each state. These measures are termed state power. To assess maturational changes in the HMM states, we plotted state power, fractional occupancy, lifetime and interval against age and fitted the resulting curve using the models outlined above. Statistical significance was determined using the same permutation approach described above, with correction for multiple comparisons achieved using a Bonferroni approach.

\section{Spatial relations between HMM states and stationary connectivity}

Finally, we tested for any significant relationship between the state maps and the spatial distribution of stationary connectivity change with age. To achieve this we first summed the age-connectivity matrix, in one dimension, to generate a $1 \times 78$ vector highlighting which brain regions showed the strongest maturational effects. Pearson correlation between this vector and each of the state maps was then computed. It is important to note that statistical significance of the Pearson correlation is non-trivial; this is because the number of degrees of freedom across the 78 brain regions is unknown (to assume 78 would likely be an over-estimate since it would fail to account for spatial blurring across AAL 
regions). Statistical significance was therefore computed using an empirical pseudo-matrix approach (Hunt et al. 2016; Tewarie et al. 2016). Briefly, a phase randomisation procedure was used to generate a fake matrix, similar in spatial smoothness to the real age connectivity matrix but containing no genuine information. This was then summed in one dimension to generate a sham vector and Pearson correlation of this with the real HMM state maps calculated. Multiple $(100,000)$ realisations of this process generated empirical null distributions against which the real spatial correlation could be tested. In this way, empirical $p$-values were derived; statistical significance was defined as $p<0.05$ corrected for multiple comparisons across HMM states using the Bonferroni method.

\section{RESULTS:}

Neural oscillatory amplitude, stationary connectivity strength, and age

Global (whole brain) functional connectivity strength is plotted against age in Figure 1A. Global neural oscillatory amplitude is plotted against age in Figure 1B. In agreement with our hypothesis (i), relationships between global connectivity strength and age are observed in the alpha and beta bands, with older participants exhibiting higher connectivity. In both cases, a non-linear-monotonic model, in which the rate of increase slowed with increasing age, gave the best fit to the data. The fit is shown by the yellow lines in Figure $1 \mathrm{~A}$, and the modulation was statistically significant ( $p_{n / m}=0.0006$ for alpha and $p_{n / m}=0.0048$ for beta) compared to an empirical null distribution. (A linear model also demonstrated a significant increase in connectivity with age. A second order polynomial fit (with negative quadratic term, used to test for a mid-age-range peak) failed to yield a significant result.) These findings show that coordination of alpha and beta oscillations among spatially separate brain regions increases significantly with maturation. The fact that these changes occur in the $8-30 \mathrm{~Hz}$ range is consistent with work showing that connectivity in a number of resting state networks is mediated within these bands (Brookes, Woolrich, et al. 2011; Hipp et al. 2012). It is noteworthy that removal of outliers had no effect on these results (see Appendix 1 ). A significant ( $p_{\text {quadratic }}=0.0008$ ) quadratic relationship between connectivity and age was observed in the theta band, with connectivity increasing throughout childhood, peaking around age 18, and falling in participants in their early 20 s.

Figure 1B shows a negative linear trend between age and theta band oscillatory amplitude ( $p_{\text {linear }}=$ 0.018), with older participants exhibiting lower amplitude. This has been observed in previous work (Clarke et al. 2001; Gasser et al. 1988; Schäfer et al. 2014) although here the finding was not significant after multiple comparison correction. A quadratic relationship $\left(p_{\text {quadratic }}=0.026\right)$ was observed between alpha amplitude and age, with amplitude peaking around age 18 years. Beta band amplitude also mirrored this pattern, but the relationship failed to reach statistical significance. Importantly, these alpha and beta band results show a clear demarcation between our amplitude and connectivity data, with the monotonic increase in connectivity in the alpha and beta bands (Figure $2 \mathrm{~A}$ ) not mirrored by amplitude changes. (We further showed that there was no measurable spatial relationship between amplitude 
and connectivity change (see SI Figure S3)). Gamma amplitude showed a general trend towards an increase with age, however this failed to reach significance. 

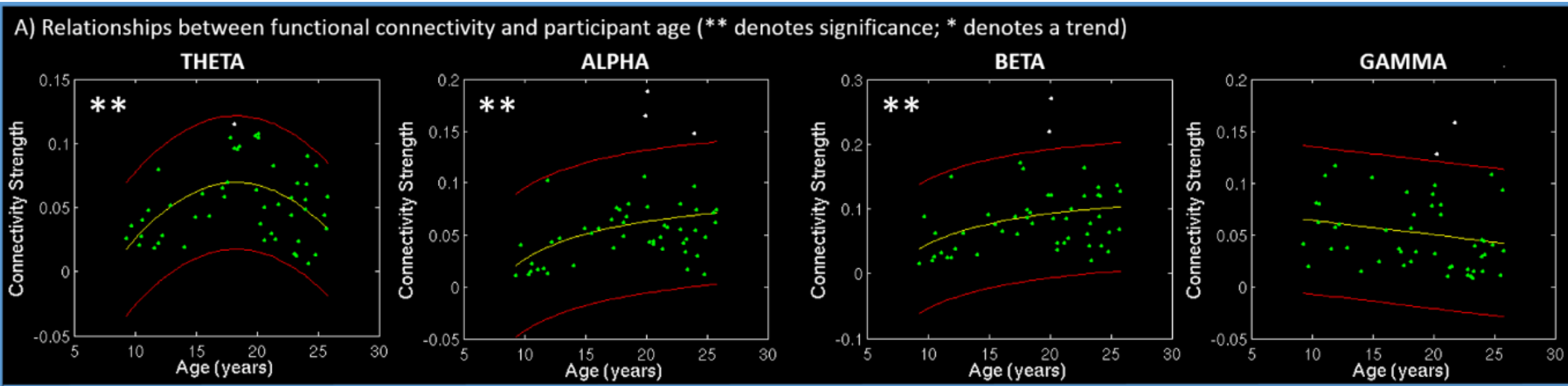

B) Relationships between neural oscillatory amplitude and participant age (** denotes significance; ${ }^{*}$ denotes a trend)
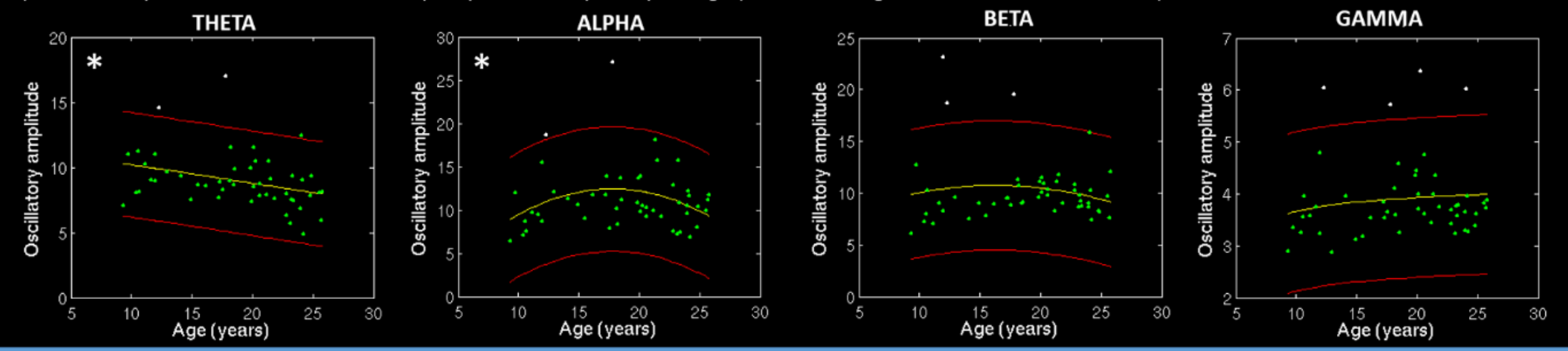

Figure 1: The relationship between neural oscillatory amplitude, connectivity strength and age at the whole brain level. A) Global connectivity strength (summed across all connections in the brain) computed for all participants and plotted against age for four frequency bands, theta $(4-8 \mathrm{~Hz})$, alpha $(8-13 \mathrm{~Hz})$, beta $(13-35 \mathrm{~Hz})$ and gamma $(35-7 \mathrm{~Hz})$. Significant ( $p<0.05$ corrected for multiple comparisons) non-linear-monotonic increases in connectivity were observed in the alpha and beta bands and a significant quadratic relationship was observed in the theta band. B) Mean oscillatory amplitude (collapsed across all 78 AAL regions) for all participants plotted as a function of age. A negative linear trend between theta amplitude and age was observed, alongside a quadratic trend in the alpha band; in both cases the finding itself was significant ( $p<0.05$ ) but did not survive multiple comparison correction across frequency bands. In both A) and B) the yellow overlays show best fitting curves (see equations $1-3$ ). Red curves show plus/minus 2 standard deviations from the model fit; points in white are statistical outliers. Note that statistics were computed with and without inclusion of those outliers - see Appendix 1: Tables A1 and A2. 
The spatial morphology of stationary connectivity change with age

Figures $2 \mathrm{~A}$ and $2 \mathrm{~B}$ show age-connectivity-matrices in the beta and alpha bands respectively. The matrices show the magnitude of the age-connectivity relationship mapped across region pairs (i.e. red indicates a region pair that changes markedly with age; green would imply no change with age). The glass brain allows visualisation of this matrix in brain space, with the lines showing the $5 \%$ of connections that change most (and significantly) between mid-childhood and early-adulthood. Line thickness and colour represent the magnitude of the relationship. In agreement with our hypothesis (ii), the change in global connectivity strength is spatially specific: In the beta band, maturation is dominated by changing connectivity between the left and right parietal regions and changing temporo-parietal connections. (Though it is noteworthy that some fronto-parietal connectivity differences are also observed). In the alpha band, the spatial profile of connectivity change is similar, again being dominated by bilateral parietal and temporal regions (note that the visual system shows comparatively little modulation with age). Figure 2C shows a spatial mapping of connectivity change in the theta band. Recall that, unlike alpha and beta, theta modulation with age follows a quadratic pattern (see Figure 1), in which connectivity increases from age 9 to 18 and subsequently decreases. Spatially this is most strongly expressed in connections between parietal and temporal cortices, which is similar to the spatial signatures of alpha and beta band modulation. These findings were mirrored by group comparisons (i.e. contrasting older to younger subjects) and by seed based connectivity assessment (these supplementary results are shown in Figures S4 and S5 respectively).

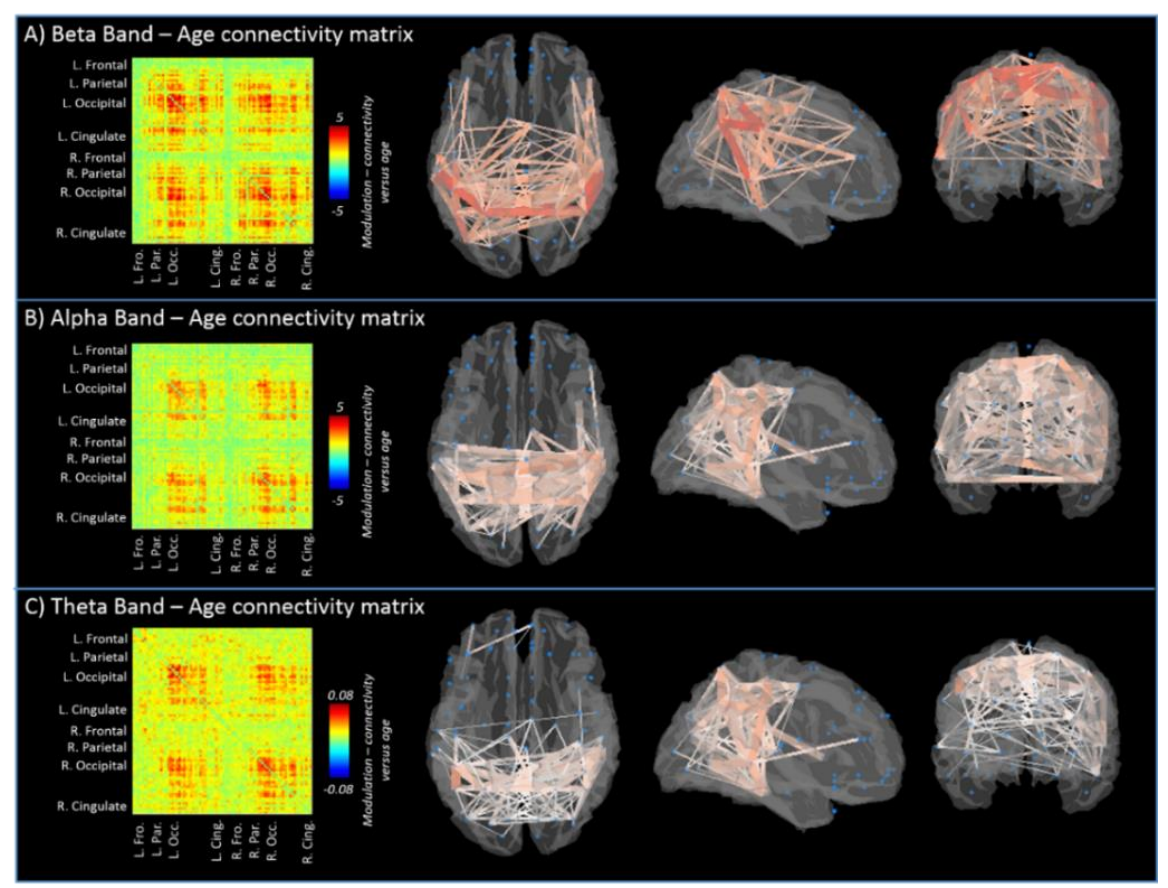

Figure 2: The spatial signature of the age-connectivity-relationship: A) Age-connectivity matrix for the beta band. The matrix depicts the fitted parameter, $b_{1}$ (see Methods), which represents the magnitude of the relationship for all possible connections. The glass brain images show a visualisation of this matrix. The line thickness and colour denotes relationship strength, with the top $5 \%$ of age-connectivity gradients visualised. $B$ ) Equivalent to $(A)$ but depicts the alpha band age-connectivity relationship. C) Equivalent to $(A)$ and (B) but for the theta band. Here the matrix represents the magnitude of the quadratic relationship between age and connectivity (see Figure 1) (i.e. each matrix element represents model standard deviation std quad. $_{\text {. }}$ ) 
Hidden Markov Model (HMM) shows that rapid network dynamics change with age Using our HMM we aimed to determine whether altered dynamics within transient brain networks were underlying the stationary connectivity differences shown in figure 2 . With this in mind, a HMM (Baker et al. 2014) was applied to parcellated MEG data. We derived 8 HMM brain 'states' (where a 'state' represents a unique spatial pattern of oscillatory envelope amplitudes and amplitude couplings that consistently repeats at different points in time). Figures $3 \mathrm{~A}-\mathrm{H}$ show the inferred HMM states. In all cases the state map shows the brain regions involved (derived as the partial correlation of the state timecourse with the amplitude envelope timecourse, concatenated across subjects). Red represents brain areas whose amplitude envelope increases when the brain visits that state; blue represents brain areas whose amplitude envelope decreases. (In the visualisation, the cortex is divided into the $78 \mathrm{AAL}$ parcels, and each region is evenly shaded.) Despite the large age range used in this study, the state maps are in good agreement with those defined by Baker and colleagues (Baker et al. 2014), with states representing the visual and sensorimotor systems as well as the bilateral temporal, frontal and parietal cortices. The inset scatter plots show state power (averaged over all parcels) as a function of age across participants. States marked ${ }^{* *}$ demonstrated a significant non-linear monotonic change in power with age $(p<0.05$ following multiple comparison correction allowing for 8 states - see als Appendix 1, Table A3). For illustrative purposes, Figures 3I-K show the fractional occupancy $(\mathrm{I})$, state lifetime $(\mathrm{J})$ and interval time $(\mathrm{K})$ averaged across all subjects. Note that the networks depicted by the state maps are shown to switch on and off on a time scale of $\sim 50$ $100 \mathrm{~ms}$ - this is in agreement with Baker et al (2014). 

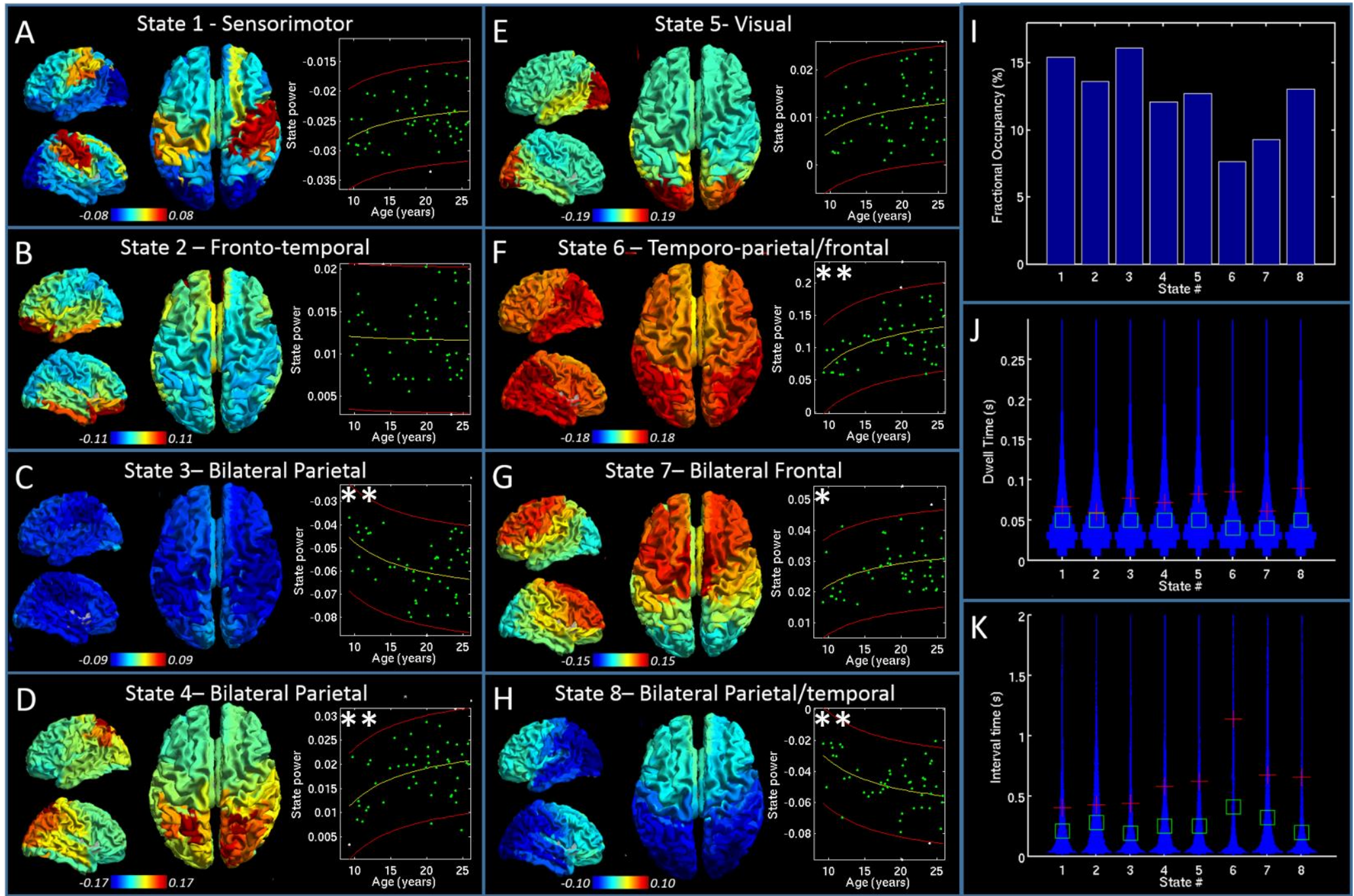

Figure 3: HMM state maps and statistics. Panels A - H show subject average HMM state maps. Red represents brain regions with increased power relative to average. Likewise blue represents brain regions with decreased power. The scatter plots show state power as a function of subject age. Statistical outliers are shown in white; red lines show twice the standard deviation around the model fit. Note significant age relationships in states 3, 4, 6 and 8 (with and without inclusion of outliers), all of which capture brain regions implicated by our stationary connectivity analysis in Figure 2. A trend is noted in state 7. (See also Appendix 1, Table A3.) Panels I, J and K show the fractional occupancy (measured across all subjects), state lifetime (distribution across subjects) and interval between state visits (distribution across subjects) for each of the 8 states respectively. Note that the state maps and statistics are in good agreement with Baker et al, elife, 2014. 


\section{The relationship between stationary connectivity and HMM states}

Of the 8 states inferred, 4 demonstrated significant power change with age, and it is of note that these 4 shared some spatial overlap with the brain regions in which stationary functional connectivity was changing the most, specifically bilateral parietal and temporal cortices (See Figure 2). This, qualitatively, lends support to our hypothesis (iii) that changing network dynamics underlie changes in stationary functional connectivity.

To test this quantitatively, we first computed the integral, in one dimension, of the age-connectivity matrices shown in Figure 2 (i.e. we summed the matrices over all rows). The result is a $1 \times 78$ vector which shows the average change in connectivity with age, between each region and all other AAL parcels. We term these measures the age-connectivity-vectors and they are shown, visualised on the cortical surface, in Figures 4A (alpha band) and 4B (beta band). Red regions represent brain areas whose connectivity to all other brain areas increases the most with age. Blue regions show little age related change. As expected from Figure 2, for both the alpha and beta bands, these maps are dominated by maturational effects in lateral parietal and temporal regions, with some effects in the frontal lobes.

Having computed the age connectivity vectors, we assessed Pearson correlation (over regions) between those vectors (averaged across the alpha and beta bands) and all 8 state maps shown in Figure 3. This revealed that state 6 - the temporo/parietal/frontal state - demonstrates a significant $(p<0.05)$ relationship with the spatial signature of stationary functional connectivity change with age. Figure $4 \mathrm{C}$ shows the spatial map associated with this state (this is equivalent to the map in Figure 3F but has been windowed for easier comparison) and the significant agreement between this and the age connectivity vectors in Figures 4A and 4B can be seen visually. Interestingly, this state has the lowest fractional occupancy, and the highest interval time between visits, consistent with what Baker et al termed the 'Default Mode' state. The three inset panels in Figure 4C show fractional occupancy, state lifetime and interval between state visits for as a function of age. We observe that both fractional occupancy and mean state lifetime increase significantly with age, whilst the interval between states shows no measurable effect. This implies that visits to this state, which captures brain regions commonly associated with attention and cognition, tend to last longer in older subjects compared to younger subjects. The spatial agreement between stationary connectivity and this state map, coupled with our finding of increased state lifetimes, confirms our hypothesis (iii) that changes in stationary functional connectivity with age are underpinned by changes in rapid network dynamics: specifically, as the brain matures, it tends to spend more time within this cognitive/attentional state and this is likely to drive an increase in functional connectivity between associated regions. 


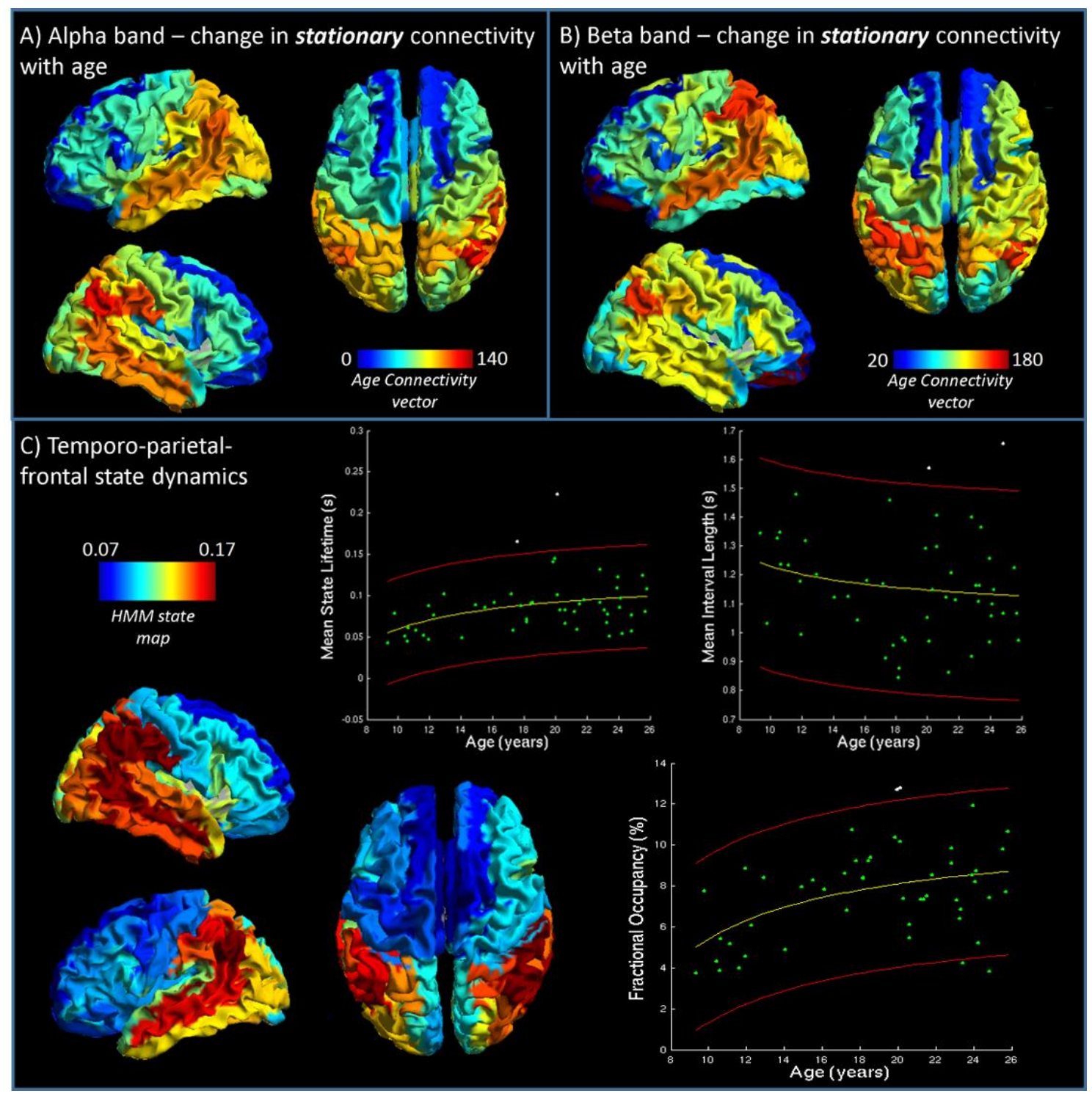

Figure 4: Temporo-parietal/frontal State: Statistics and comparison with stationary functional connectivity. A) Brain regions in which stationary functional connectivity changes the most with age in the 9-25 year range in the alpha band (computed as the sum, in one dimension, of the matrix shown in Figure 2B). B) Equivalent to (A) but for the beta band. C) Shows again the spatial map for state 6 - note that this has been rescaled compared to that shown in figure 3 . The three inset scatter plots show fractional occupancy, mean state lifetime and mean interval between state visits as a function of age. The yellow curves show a non-linear monotonic model (Equation 2) fitted to the data. Statistical outliers are shown in white; red lines show twice the standard deviation around the model fit. Significant change with age was observed for fractional occupancy and state lifetime (with and without inclusion of outliers); no change in interval between state visits with age was measurable. (See also Appendix 1: Table A4)

\section{DISCUSSION:}

The brain regions whose static functional connectivity changes most, between mid-childhood and early adulthood, included the left and right superior and inferior parietal lobules, bilateral intraparietal sulci, bilateral angular and supramarginal gyri and bilateral temporal cortices. In addition, development of connections between parietal regions and the frontal lobes was also observed (see also Figure S5). These regions exhibit substantial overlap with the structural and functional "backbone" identified by Hagmann et al. (Hagmann et al. 2008). This backbone extends from a core in medial and lateral parietal cortex, via the temporo-parietal junction, into the lateral temporal lobe 
and also to the frontal lobes. This set of regions is highly connected both structurally and functionally, and is likely to play a central role in enabling efficient communication between other functionally specific regions. In a MEG study of adults, Tewarie et al (Tewarie P.K. et al. 2014) demonstrated that the pattern of connectivity in the alpha and beta bands closely resembled the pattern of connectivity assessed using resting state $\mathrm{fMRI}$, within the parietal and temporal regions associated with this backbone. Further, in a developmental study (Hagmann et al. 2010), which used diffusion tensor imaging to assess structural connectivity and fMRI to assess functional connectivity, centrality rank (which reflects the role of a network node in mediating connections between other pairs of nodes) was already well developed in the backbone in early childhood but correlation between structural and functional connectivity increased significantly with age up to 18 years. This change over development in network properties indicates age related increase in integration across the brain, and concomitant decrease in segregation between localized modules. Hagmann et al. (2010) proposed that such increasing integration would be related to neural synchrony. This hypothesis is in accord with our observations and we speculate that our findings of increased connectivity represent the development of this backbone of structural and functional connectivity, and thus increasing integration across brain regions with age.

Our hidden Markov model is, to our knowledge, the first dynamic electrophysiological network analysis in a neurodevelopmental cohort. The HMM allowed derivation of 8 dynamic networks, the spatial and temporal signatures of which are in strong agreement with previous work in an adult population using the same method (Baker et al. 2014). These include the visual and sensorimotor networks as well as networks that capture brain areas classically associated with attention and cognition. Our analysis of state power shows that four of the 8 derived states modulate significantly with age, and all of these capture either bilateral parietal cortex (states 3 and 4) or bilateral temporal and parietal regions (states 6 and 8). Of these four states, one (state 6) exhibited significant spatial similarity when compared to stationary connectivity change with age. Its spatial morphology, coupled with low state occupancy and high interval between state visits (shown in Figures $3 \mathrm{l}$ and $\mathrm{K}$ respectively) suggests that this state is equivalent to what Baker and colleagues termed the default mode. Our results show that as subjects age their brains tend to spend more time in this state (increased fractional occupancy). It is striking that this increase in fractional occupancy is driven by an increase in state lifetimes, with no apparent decrease in interval time (see Figure 4). This suggests that the change with age is specific to the network being more stable once it is activated (as distinct from entering the state more often). The link (based on morphology) between this state and attentional processes suggests the relatively slow evolution of state dynamics with increasing age might underlie the protracted nature of the development of the attentional system. This finding paves the way for future research assessing the relationship between state dynamics and behaviour. 
The fact that using two separate analyses, the first a "classical" functional connectivity analysis and the second an assessment of dynamic states, we were able to derive significant changes with age in the same cortical regions is compelling evidence, not only of maturational change in these regions, but also of a link between the HMM state dynamics and stationary functional connectivity. A similar link had been reported previously, albeit in an adult population. In Baker et al. (Baker et al. 2014), the authors showed that in healthy adult subjects, time windows in which state visits were more likely resulted in a higher functional connectivity between the regions associated with that state. Our finding agrees with this observation in showing that, as visits to the attentional state become more stable in time, connectivity between the same brain regions increases. It is important to note that the existence of a spatial overlap does not necessarily imply a causal relation between state dynamics and stationary connectivity, nevertheless it is tempting to speculate that as the brain matures increased temporal stability in core attentional areas drives increased functional connectivity.

The relationship between connectivity change with age, and amplitude change with age, warrants further discussion. In all bands, connectivity changes with age differed from changes in amplitude. In the alpha and beta bands, connectivity increased monotonically whereas amplitude showed a peak in the late teenage years (though this was not significant). Conversely, in the theta band, connectivity showed a peak, whilst amplitude showed a trend for a linear monotonic decrease. This demarcation between the developmental trajectory of connectivity and amplitude implies that the observable connectivity increases with age are not simply a result of SNR changes but reflect genuine increases in the extent to which oscillations are coordinated across the brain. This was supported by further findings (see Figure S3) showing that in both the theta and alpha bands (i.e. those bands in which amplitude was trending - see Figure 1) there was no significant spatial overlap between amplitude and connectivity changes (i.e. even if there was a trend in amplitude in some brain regions, it had no bearing on the spatial signature of connectivity change). It is not clear why oscillatory amplitude in the alpha and beta bands should show a developmental peak. However, it has been established that cortical and white matter myeloarchitecture is altered by electrical activity (Gibson 2014). Furthermore, electrophysiological connectivity and cortical myeloarchitecture display correlations in an adult population (Hunt et al. 2016). Given this, it is plausible that a transient increase in alpha and beta band neural oscillatory amplitude in the teenage years might mediate the formation of long range alpha and beta band connections between regions, via manipulation of structural morphology (specifically myeloarchitecture). However, such a theory remains speculation and, if this were the case, we might expect a spatial relationship between the transient increase in alpha amplitude and the monotonic increase in alpha connectivity, which we did not observe. Further work is therefore required to establish the existence of such a mechanism. The quadratic relationship of theta band connectivity also remains an open-ended question. However, data reported by Schäfer et al (Schäfer et al. 2014) which strongly supported our alpha and beta band findings, failed to show a similar quadratic effect in the theta band. We therefore advise caution when interpreting this result. 
Finally, the reader should be aware of a number of inherent limitations of our study. First, our sample of 50 participants was relatively small, and repeating the current analyses in a larger population is an area for future work. Second, in recruitment we attempted to ensure no systematic effect of IQ with age. Nonetheless, a weak but statistically significant positive correlation between age and IQ existed in our final sample, raising the possibility that our age effects may have been confounded by IQ. In supplementary analyses (see SI Figure S6 and Tables S3 and S4) we addressed this by including the IQ measurements as an effect of no interest in our general linear model fit. Results showed that even with IQ included in the regression, the primary effect of age was still significant; moreover there was no significant relationship between IQ and either connectivity or amplitude, in any frequency band. Third, we used relatively broad frequency bands in our analysis, particularly for beta and gamma ranges. There is an inherent $1 / f$ distribution of oscillatory power in the electrophysiological signal, and this means that broader frequency bands tend to be dominated by their lower end (see SI Figure S7). In principle the use of an increased number of narrower bands may be desirable, however this would be very computationally intensive. Fourth, an inherent limitation is that, since magnetic field falls with the square of distance from the source, MEG exhibits lower sensitivity to deeper sources. In the context of the present study this means that deeper cortical AAL regions exhibit lower signal to noise ratio and hence connectivity among such regions may be artefactually diminished - at the time of writing no correction for such SNR loss is possible. Fifth, the only predictor of connectivity and oscillatory power we considered other than age was IQ. However, much of the variation in the data could also be accounted for by other individual differences between participants (e.g. it has been shown that connectivity changes significantly based on, for example, working memory abilities (Astle et al. 2015)). It is therefore important that future studies should use a multi-variate model in which multiple explanatory variables are employed to predict changing connectivity throughout development. However a larger study with more individuals would be required to obtain the necessary statistical power to support more complex models. A final limitation of the current study is the age range studied. Our youngest participants were nine years old, an age at which many functions (e.g. movement, vision) are already largely mature. Realisation of the full utility of electrophysiological connectivity to understand neurodevelopment would require scanning much younger participants. This would not only allow investigation of basic systems but would also increase the clinical utility of such investigation (e.g. disorders such as autism manifest in children as young as two). However, an inherent limitation of commercially available MEG systems is that cryogenic sensor locations are fixed in position, meaning that high SNR data acquisition and whole head coverage in very young participants is challenging due to their smaller head size. For this reason, future studies should look to non-cryogenic systems (e.g. (Boto, Bowtell, et al. 2016; Boto, Meyer, et al. 2016)), in which a single MEG instrument can be used across all age groups, from babies to adults with no instrumental confounds, to capture the developmental trajectory of neural networks across a clinically relevant age range. 


\section{CONCLUSION:}

We have shown that stationary functional connectivity (measured via inter-regional coordination of neural oscillations) increases with age in the alpha and beta bands, in bilateral parietal and temporoparietal connections. Further, a dynamic connectivity analysis on the same data (using a HMM applied to alpha/beta band oscillations) revealed the spatiotemporal signatures of 8 dynamic networks; these modulate rapidly in time, and temporal stability in networks capturing attentional regions was found to increase with age. Finally, significant overlap was found between agemodulated dynamic networks and inter-regional oscillatory coordination, implying that altered network dynamics underlie age related changes in stationary connectivity. Our results provide new insights into developmental change in human electrophysiology, and lay a foundation for future work in which childhood disorders are characterised by abnormal maturational trajectory of stationary and dynamic neural networks.

\section{APPENDIX 1: Statistical tables}

The tables below show regression parameters and associated statistics (with and without outlier removal) corresponding to the curve fits in Figures 1, 3 and 4. Table A1 shows the case for variation of functional connectivity with age. Table A2 shows the case for variation of oscillatory amplitude with age. Table A3 shows the case for state power variation with age (from Figure 3 ) and Table A4 shows the case for state 6 statistical parameters (Figure 4). In all 4 cases, for parameter definition see Equations 1, 2 and 3. The central column (labelled basic regression) shows the case where all of the data are used. The right hand column (labelled Regression with outliers removed) shows the case where the outliers (denoted by the white points in Figures 11,3 and 4) have been excluded from the statistical analysis. ${ }^{* *}$ denotes statistical significance after multiple comparison correction; * denotes a trend (i.e. significant before multiple comparison correction). Note that removal of outliers has no influence on statistical significance of our final results.

\begin{tabular}{|c|c|c|c|c|c|c|c|c|c|}
\hline \multicolumn{10}{|c|}{ Functional connectivity variation with maturation } \\
\hline \multirow[b]{2}{*}{ Test } & \multirow[b]{2}{*}{ Model } & \multicolumn{4}{|c|}{ Basic Regression } & \multicolumn{4}{|c|}{ With Outliers Removed } \\
\hline & & $\begin{array}{l}\text { Parameter } \\
\text { values }\end{array}$ & $\mathbf{R}^{2}$ & $\mathbf{P}$ & $\begin{array}{l}\text { Comparis } \\
\text { ons }\end{array}$ & $\begin{array}{c}\text { Parameter } \\
\text { Values }\end{array}$ & $\mathrm{R}^{2}$ & $\mathbf{p}$ & $\begin{array}{c}\text { Comparis } \\
\text { ons }\end{array}$ \\
\hline $\begin{array}{c}\text { Theta } \\
\text { Connectivity } \\
\text { with age }\end{array}$ & Quadratic & $\begin{array}{l}c_{1}=-0.001 \\
c_{2}=0.024 \\
c_{3}=-0.15\end{array}$ & 0.234 & $0.0008^{* *}$ & 12 & $\begin{array}{l}c_{1}=-0.001 \\
c_{2}=0.022 \\
c_{3}=-0.132\end{array}$ & 0.214 & $0.001^{* *}$ & 4 \\
\hline $\begin{array}{c}\text { Alpha } \\
\text { Connectivity } \\
\text { with age }\end{array}$ & $\begin{array}{l}\text { Non-Linear } \\
\text { Monotonic }\end{array}$ & $\begin{array}{l}b_{1}=0.74 \\
b_{2}=0.10\end{array}$ & 0.15 & $0.0006 * *$ & 8 & $\begin{array}{l}b_{1}=0.55 \\
b_{2}=0.08\end{array}$ & 0.19 & $0.0006^{* *}$ & 4 \\
\hline $\begin{array}{c}\text { Beta } \\
\text { Connectivity } \\
\text { with age }\end{array}$ & $\begin{array}{l}\text { Non-Linear } \\
\text { Monotonic }\end{array}$ & $\begin{array}{l}b_{1}=0.95 \\
b_{2}=0.14\end{array}$ & 0.12 & $0.0044 * *$ & 8 & $\begin{array}{l}b_{1}=0.80 \\
b_{2}=0.13\end{array}$ & 0.14 & $0.0036^{* *}$ & 4 \\
\hline $\begin{array}{c}\text { Gamma } \\
\text { Connectivity } \\
\text { with age }\end{array}$ & Linear & $\begin{array}{l}a_{1}=-0.31 \\
a_{2}=0.03\end{array}$ & 0.037 & 0.18 & 8 & $\begin{aligned} a_{1} & =-0002 \\
a_{2} & =0.08\end{aligned}$ & 0.08 & 0.052 & 4 \\
\hline
\end{tabular}

Table A1: Model fit parameters and statistics associated with functional connectivity variation with age. 


\begin{tabular}{|c|c|c|c|c|c|c|c|c|c|}
\hline \multicolumn{10}{|c|}{ Oscillatory amplitude variation with maturation } \\
\hline \multirow[b]{2}{*}{ Test } & \multirow[b]{2}{*}{ Model } & \multicolumn{4}{|c|}{ Basic Regression } & \multicolumn{4}{|c|}{ With Outliers Removed } \\
\hline & & $\begin{array}{c}\text { Parameter } \\
\text { values }\end{array}$ & $\mathbf{R}^{\mathbf{2}}$ & $\mathbf{P}$ & $\begin{array}{c}\text { Compariso } \\
\text { ns }\end{array}$ & $\begin{array}{c}\text { Parameter } \\
\text { Values }\end{array}$ & $\mathbf{R}^{\mathbf{2}}$ & $\mathbf{p}$ & $\begin{array}{c}\text { Comparis } \\
\text { ons }\end{array}$ \\
\hline $\begin{array}{c}\text { Theta } \\
\text { Amplitude } \\
\text { with age }\end{array}$ & Linear & $\begin{array}{l}a_{1}=-0.14 \\
a_{2}=11.6\end{array}$ & 0.11 & $0.018^{*}$ & 8 & $\begin{array}{l}a_{1}=-0.11 \\
a_{2}=10.7\end{array}$ & 0.11 & $0.048^{*}$ & 4 \\
\hline $\begin{array}{c}\text { Alpha } \\
\text { Amplitude } \\
\text { with age }\end{array}$ & Quadratic & $\begin{array}{c}c_{1}=-0.05 \\
c_{2}=1.76 \\
c_{3}=-3.21\end{array}$ & 0.09 & $0.026^{*}$ & 12 & $\begin{array}{c}c_{1}=-0.05 \\
c_{2}=1.76 \\
c_{3}=-3.21\end{array}$ & 0.07 & 0.1 & 4 \\
\hline $\begin{array}{c}\text { Beta } \\
\text { Amplitude } \\
\text { with age }\end{array}$ & Quadratic & $\begin{array}{c}c_{1}=-0.18 \\
c_{2}=0.57 \\
c_{3}=6.06\end{array}$ & 0.02 & 0.4 & 12 & $\begin{array}{c}c_{1}=-0.003 \\
c_{2}=0.20 \\
c_{3}=7.19\end{array}$ & 0.05 & 0.8 & 4 \\
\hline $\begin{array}{c}\text { Gamma } \\
\text { Amplitude } \\
\text { with age }\end{array}$ & $\begin{array}{l}\text { Non-linear } \\
\text { Monotonic }\end{array}$ & $\begin{array}{l}b_{1}=5.40 \\
b_{2}=4.20\end{array}$ & 0.018 & 0.352 & 8 & $\begin{array}{l}b_{1}=5.03 \\
b_{2}=4.00\end{array}$ & 0.05 & 0.12 & 4 \\
\hline
\end{tabular}

Table A2: Model fit parameters and statistics associated with variation of oscillatory amplitude with age.

\begin{tabular}{|c|c|c|c|c|c|c|c|c|c|}
\hline \multicolumn{10}{|c|}{ State power: variation with age } \\
\hline & & \multicolumn{4}{|c|}{ Basic Regression } & \multicolumn{4}{|c|}{ With Outliers Removed } \\
\hline Test & Model & $\begin{array}{c}\text { Parameter } \\
\text { values }\end{array}$ & $\mathbf{R}^{2}$ & $\mathbf{P}$ & $\begin{array}{c}\text { Comparis } \\
\text { ons }\end{array}$ & $\begin{array}{c}\text { Parameter } \\
\text { Values }\end{array}$ & $\mathbf{R}^{2}$ & p & $\begin{array}{c}\text { Comparis } \\
\text { ons }\end{array}$ \\
\hline State 1 & $\begin{array}{l}\text { Non-Linear } \\
\text { Monotonic }\end{array}$ & $\begin{array}{l}b_{1}=0.069 \\
b_{2}=-0.02\end{array}$ & 0.09 & $0.03^{*}$ & 8 & $\begin{array}{l}b_{1}=0.06 \\
b_{2}=-0.02\end{array}$ & 0.1 & $0.02^{*}$ & 8 \\
\hline State 2 & $\begin{array}{l}\text { Non-Linear } \\
\text { Monotonic }\end{array}$ & $\begin{array}{c}b_{1}=0.006 \\
b_{2}=0.01\end{array}$ & 0.0008 & 0.8 & 8 & $\begin{array}{l}b_{1}=0.007 \\
b_{2}=0.01\end{array}$ & $\begin{array}{l}0.00 \\
1 \\
\end{array}$ & 0.8 & 8 \\
\hline State 3 & $\begin{array}{l}\text { Non-Linear } \\
\text { Monotonic }\end{array}$ & $\begin{array}{l}b_{1}=-0.26 \\
b_{2}=-0.07\end{array}$ & 0.16 & $0.003^{* *}$ & 8 & $\begin{array}{l}b_{1}=-0.25 \\
b_{2}=-0.07\end{array}$ & 0.17 & $0.003^{* *}$ & 8 \\
\hline State 4 & $\begin{array}{l}\text { Non-Linear } \\
\text { Monotonic }\end{array}$ & $\begin{array}{l}b_{1}=0.14 \\
b_{2}=0.03\end{array}$ & 0.19 & $0.002 * *$ & 8 & $\begin{array}{l}b_{1}=0.10 \\
b_{2}=0.02\end{array}$ & 0.11 & 0.026 & 8 \\
\hline State 5 & $\begin{array}{l}\text { Non-Linear } \\
\text { Monotonic }\end{array}$ & $\begin{array}{l}b_{1}=0.10 \\
b_{2}=0.02\end{array}$ & 0.09 & $0.03 *$ & 8 & $\begin{array}{l}b_{1}=0.08 \\
b_{2}=0.02\end{array}$ & 0.07 & 0.064 & 8 \\
\hline State 6 & $\begin{array}{l}\text { Non-Linear } \\
\text { Monotonic }\end{array}$ & $\begin{array}{l}b_{1}=0.95 \\
b_{2}=0.17\end{array}$ & 0.22 & $0.0002^{* *}$ & 8 & $\begin{array}{l}b_{1}=0.86 \\
b_{2}=0.16\end{array}$ & 0.25 & $0.0002^{* *}$ & 8 \\
\hline State 7 & $\begin{array}{l}\text { Non-Linear } \\
\text { Monotonic }\end{array}$ & $\begin{array}{c}b_{1}=0.014 \\
b_{2}=0.04\end{array}$ & 0.11 & $0.01^{*}$ & 8 & $\begin{array}{l}b_{1}=0.11 \\
b_{2}=0.03\end{array}$ & 0.10 & $0.02 *$ & 8 \\
\hline State 8 & $\begin{array}{l}\text { Non-Linear } \\
\text { Monotonic }\end{array}$ & $\begin{array}{l}b_{1}=-0.38 \\
b_{2}=-0.07\end{array}$ & 0.18 & $0.0005^{* *}$ & 8 & $\begin{array}{l}b_{1}=-0.33 \\
b_{2}=-0.07\end{array}$ & 0.19 & $0.002^{* *}$ & 8 \\
\hline
\end{tabular}

Table A3: Model fit parameters and statistics associated with HMM state power variation with age.

\begin{tabular}{|c|c|c|c|c|c|c|c|c|c|}
\hline \multicolumn{10}{|c|}{ State 6: model parameters } \\
\hline \multirow[b]{2}{*}{ Test } & \multirow[b]{2}{*}{ Model } & \multicolumn{4}{|c|}{ Basic Regression } & \multicolumn{4}{|c|}{ With Outliers Removed } \\
\hline & & $\begin{array}{l}\text { Parameter } \\
\text { values }\end{array}$ & $\mathbf{R}^{2}$ & $\mathbf{P}$ & $\begin{array}{l}\text { Comparis } \\
\text { ons }\end{array}$ & $\begin{array}{l}\text { Parameter } \\
\text { Values }\end{array}$ & $\mathbf{R}^{2}$ & $\mathbf{p}$ & $\begin{array}{c}\text { Comparis } \\
\text { ons }\end{array}$ \\
\hline $\begin{array}{l}\text { Fractional } \\
\text { Occupancy }\end{array}$ & $\begin{array}{l}\text { Non-Linear } \\
\text { Monotonic }\end{array}$ & $\begin{array}{l}\mathrm{b}_{1}=53.8 \\
\mathrm{~b}_{2}=10.8\end{array}$ & 0.21 & $0.0012^{* *}$ & 4 & $\begin{array}{l}b_{1}=49.3 \\
b_{2}=10.3\end{array}$ & 0.22 & $0.0016^{* *}$ & 4 \\
\hline $\begin{array}{l}\text { Mean state } \\
\text { lifetime }\end{array}$ & $\begin{array}{l}\text { Non-Linear } \\
\text { Monotonic }\end{array}$ & $\begin{array}{l}b_{1}=0.64 \\
b_{2}=0.12\end{array}$ & 0.14 & $0.0028^{* *}$ & 4 & $\begin{array}{l}b_{1}=0.57 \\
b_{2}=0.12\end{array}$ & 0.21 & $0.0004^{* *}$ & 4 \\
\hline $\begin{array}{c}\text { Mean } \\
\text { interval }\end{array}$ & $\begin{array}{l}\text { Non-Linear } \\
\text { Monotonic }\end{array}$ & $\begin{array}{c}b_{1}=-0.17 \\
b_{2}=1.1\end{array}$ & 0.03 & 0.24 & 4 & $\begin{array}{l}b_{1}=-2.4 \\
b_{2}=1.00\end{array}$ & 0.08 & $0.048^{*}$ & 4 \\
\hline
\end{tabular}

Table A4: Model fit parameters and statistics associated with HMM state 6 variation with age. 


\section{APPENDIX 2: Muscle artifacts and their effect on connectivity}

Muscle artifacts comprise the unwanted magnetic fields that are generated by electrophysiological activity in muscles, in the head and neck. They span a wide frequency range, beginning in the beta band $(\sim 20 \mathrm{~Hz})$ and extending (and becoming stronger) up to the high gamma band (Claus et al. 2012; Muthukumaraswamy 2013; Whitham et al. 2007; Zimmermann and Scharein 2004 ). Critically, muscle artifacts tend to effect many sensors, and often appear bilaterally (e.g. tensing the muscles in the jaw leads to increased oscillatory amplitude in sensors on both the left and right of the head). Such effects, in principle, could spuriously increase metrics of functional connectivity made using amplitude envelope correlation.

In the present paper, we addressed this problem by first making two assumptions: i) In resting state data, the $120-150 \mathrm{~Hz}$ band contains no useful neuromagnetic information, but is sensitive to muscle activity. ii) The envelope of muscle artifact in the $120-150 \mathrm{~Hz}$ range is the same as the envelope of the equivalent artifact in the lower frequency bands. Given these two assumptions, it is possible to reduce the muscle artifact by frequency filtering the beamformer projected data into the $120-150 \mathrm{~Hz}$, computing the oscillatory envelope in this high frequency band, and regressing that envelope from the equivalent envelope at lower frequencies. This technique has been adopted in previous work (Brookes et al. 2016; Liuzzi et al. 2016).

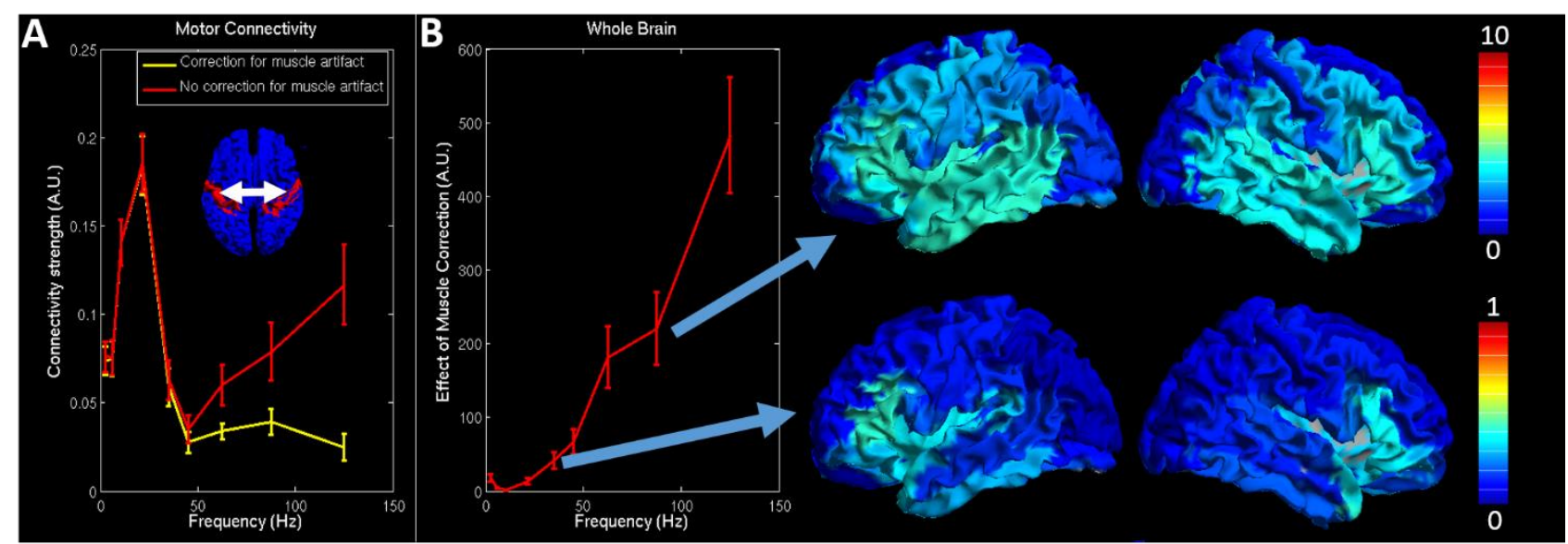

Figure A1: The effect of muscle artifact reduction. A) The magnitude of functional connectivity, with (yellow) and without (red) muscle artifact reduction, between the left and right primary sensory cortex, plotted as a function of frequency. B) The graph shows the difference in connectivity strength, averaged across the whole brain, with and without muscle artifact reduction. The inset images show the spatial distribution of this measured difference.

Figure A1A shows the magnitude of functional connectivity between the left and right primary sensorimotor cortex, plotted as a function of frequency. The red curve shows the case with no reduction of the muscle artifact via the regression method, whilst the yellow curve shows the case with correction for muscle artifact. Note that in the absence of the correction, there is a marked 
increase in functional connectivity with frequency in the $>50 \mathrm{~Hz}$ range. However with reduction applied, this increase is lost and functional connectivity across the gamma band remains constant. The likely conclusion is that this increase is artefactual; it is driven entirely by muscle artifacts but is well controlled by our regression methodology. In Figure A1B, the graph shows the difference in whole brain connectivity strength with and without muscle artifact reduction. The inset images show the spatial distribution across the brain, of this measured difference. Note that the muscle artifact primarily affects the frontal and temporal regions; this is likely consistent with activity of the muscles controlling the jaw. Importantly, such effects are relatively small for the alpha and beta bands, but become considerably more problematic in the gamma band (note the difference in scales). Whilst the present method is effective at controlling these artifacts, a more complete solution, which should be considered in future work, is to use a set of electrodes placed immediately over the muscle groups of interest such that their activity can be recorded directly, and regressed from the data.

\section{ACKNOWLEDGEMENTS:}

This work was funded by an MRC New Investigator Research Grant (MR/M006301/1) and an MRC studentship awarded to HJFS. We also acknowledge Medical Research Council Partnership Grant (MR/K005464/1). MWW is supported by the Wellcome Trust (106183/Z/14/Z) and the Wellcome Centre for Integrative Neuroimaging is supported by core funding from the Wellcome Trust (203139/Z/16/Z).

\section{REFERENCES:}

Allen, E.A., E. Damaraju, S.M. Plis, E.B. Erhardt, T. Eichele, and V.D. Calhoun. 2014. 'Tracking whole-brain connectivity dynamics in the resting state', Cereb Cortex, 24: 663-76

Astle, D.E., J.J. Barnes, K. Baker, g.L. Colclough, and M.W. Woolrich. 2015. 'Cognitive Training Enhances Intrinsic Brain Connectivity in Childhood', Journal of Neuroscience, 35 6277-83.

Baker, A. P., M. J. Brookes, I. A. Rezek, S. M. Smith, T. Behrens, P. J. P. Smith, and M. Woolrich. 2014. 'Fast transient networks in spontaneous human brain activity', eLife, 2014.

Boto, E., R. Bowtell, P. Krüger, T. M. Fromhold, P. G. Morris, S. S. Meyer, G. R. Barnes, and M. J. Brookes. 2016. 'On the potential of a new generation of magnetometers for MEG: A beamformer simulation study', PLoS One, 11.

Boto, E., S. Meyer, V. Shah, O. Alem, S. Knappe, P. Kruger, T.M. Fromhold, M. Lim, P.M. Glover, P.G. Morris, R. Bowtell, g.R. Barnes, and M. J. Brookes. 2016. 'A New Generation of Magnetoencephalography: Room Temperature Measurements using Optically-Pumped Magnetometers', Neurolmage, In Press.

Brookes, M. J., G. C. O'Neill, E. L. Hall, M. W. Woolrich, A. Baker, S. Palazzo Corner, S. E. Robson, P. G. Morris, and G. R. Barnes. 2014. 'Measuring temporal, spectral and spatial changes in electrophysiological brain network connectivity', Neurolmage, 91: 282-99.

Brookes, M. J., P. K. Tewarie, B. A. E. Hunt, S. E. Robson, L. E. Gascoyne, E. B. Liddle, P. F. Liddle, and P. G. Morris. 2016. 'A multi-layer network approach to MEG connectivity analysis', Neurolmage, 132: 42538. 
Brookes, M.J., J.R. Hale, J.M. Zumer, C.M. Stevenson, S.T. Francis, G.R. Barnes, J.P. Owen, P.G. Morris, and S.S. Nagarajan. 2011. 'Measuring Functional Connectivity Using MEG: Methodology and Comparison With fcMRI ', Neurolmage, 56: 1082-104.

Brookes, M.J., J. Vrba, S.E. Robinson, C.M. Stevenson, A.P. Peters, G.R. Barnes, A. Hillebrand, and P.G. Morris. 2008. 'Optimising experimental design for MEG beamformer imaging ', Neurolmage, 39: 1788-802.

Brookes, M.J., M. Woolrich, H. Luckhoo, D. Price, J.R. Hale, M.C. Stephenson, G.R. Barnes, S.M. Smith, and P.G. Morris. 2011. 'Investigating the Electrophysiological Basis of Resting State Networks using Magnetoencephalography', Proceedings of the National Academy of Science USA, 108: 16783-88.

Brookes, M.J., M.W. Woolrich, and G.R. Barnes. 2012. 'Measuring Functional Connectivity in MEG: a MultiVariate Approach insensitive to linear Source Leakage', Neurolmage, 63: 910-20.

Clarke, A.R., R.J. Barry, R. McCarthy, and M. Selikowitz. 2001. 'Age and sex effects in the EEG: Development of the normal child', ClinNeurophysiol, 112: 806-14.

Claus, S.C., D. Velis, F.H. Lopes da Silva, M.A. Viergever, and S. Kalitzin. 2012. 'High frequency spectral components after Secobarbital: The contribution of muscular origin-A study with MEG/EEG', Epilepsy Research, 100: 132-41.

Colclough, G.L., M.J. Brookes, S.M. Smith, and M.W. Woolrich. 2015. 'A symmetric multivariate leakage correction for MEG connectomes', Neurolmage, In Press.

de Bie, H.M.A., M. Boersma, S. Adriaanse, D.J. Veltman, A.M. Wink, S.D. Roosendaal, F. Barkhof, C.J. Stam, K.J. Oostrom, H.A. Delemarre-van de Waal, and E.J. Sanz-Arigita. 2012. 'Resting-state networks in awake five- to eight-year old children', Human Brain Mapping, 33: 1189-201.

de Pasquale, F., S. Della Penna, A.Z. Snyder, C. Lewis, D. Mantini, A. Marzetti, P. Belardinelli, L. Ciancetta, V. Pizzella, G.L. Romani, and M. Corbetta. 2010. 'Temporal dynamics of spontaneous MEG activity in brain networks', Proceedings of the National Academy of Science USA, 107: 6040-5.

de Pasquale, F., S. Della Penna, O. Sporns, G.L. Romani, and M. Corbetta. 2015. 'A Dynamic Core Network and Global Efficiency in the Resting Human Brain', Cereb Cortex, 26.

Engel, A.K., C. Gerloff, C.C. Hilgetag, and G. Nolte. 2013. 'Intrinsic Coupling Modes: Multiscale Interactions in Ongoing Brain Activity', Neuron, 80: 867 - 86.

Fries, P. . 2015. ' Rhythms for Cognition: Communication through Coherence', Neuron 88: 220-35.

Gaetz, W., M. Macdonald, D. Cheyne, and O.C. Snead. 2010. 'Neuromagnetic imaging of movement-related cortical oscillations in children and adults: age predicts post-movement beta rebound', Neurolmage, 51: 792-807.

Gasser, T., C. Jennen-Steinmetz, L. Stroka, R. Verleger, and J. M€ocks. 1988. 'Development of the EEG of school-age children andadolescents II. Topography', Electroencephalogr Clin Neuro-physiol, 69: 10009.

Gibson, E. M. et al. 2014. 'Neuronal Activity Promotes Oligodendrogenesis and Adaptive Myelination in the Mammalian Brain', Science, 344: 1252304

Grayson, D.S., and D.A. Fair. 2017. 'Development of large-scale functional networks from birth to adulthood: A guide to the neuroimaging literature.', Neurolmage, S1053-8119(17)30102-7: doi: 10.1016/j.neuroimage.2017.01.079. [Epub ahead of print].

Grimm, K.J., N. Ram, and F. Hamagami. 2011. 'Nonlinear Growth Curves in Developmental Research', Child Dev, 82: 1357-71.

Hagmann, P., L. Cammoun, X. Gigandet, R. Meuli, C.J. Honey, V.J. Wedeen, and O. Sporns. 2008. 'Mapping the Structural Core of Human Cerebral Cortex', PLoS Biol, 6: e159.

Hagmann, P., O Sporns, N. Madan, L. Cammoun, R. Pienaar, V.J. Wedeen, R. Meuli, J-P. Thiran, and P.E. Grant. 2010. 'White matter maturation reshapes structural connectivity in the late developing human brain', Proceedings of the National Academy of Science USA, 107: 19067-72.

Hall, E. L., S. E. Robson, P. G. Morris, and M. J. Brookes. 2014. 'The relationship between MEG and fMRI', Neurolmage, 102: 80-91.

Hall, E.L., M.W. Woolrich, C.R. Thomaz, P.G. Morris, and M.J. Brookes. 2013. 'Using variance information in magnetoencephalography measures of functional connectivity.', Neurolmage, 67: 203-12.

Hipp, J.F., D.J. Hawellek, M. Corbetta, M. Siegel, and A.K. Engel. 2012. 'Large-scale cortical correlation structure of spontaneous oscillatory activity', Nature Neuroscience, 15: 884-90. 
Huang, M.X., Mosher, J.C., Leahy, R.M. 1999. 'A sensor-weighted overlapping-sphere head model and exhaustive head model comparison for MEG', Physics in Medicine and Biology, 44: 423-40.

Huizinga, M.1., C.V. Dolan, and M.W. van der Molen. 2006. 'Age-related change in executive function: developmental trends and a latent variable analysis.', Neuropsychologia, 44: 2017-36.

Hunt, B.A., P.K. Tewarie, O.E. Mougin, N. Geades, D.K. Jones, K.D. Singh, P.G. Morris, P.A. Gowland, and M.J. Brookes. 2016. 'Relationships between cortical myeloarchitecture and electrophysiological networks', Proc Natl Acad Sci U S A, pii: 201608587. [Epub ahead of print].

Hutchison, R. M., T. Womelsdorf, E. A. Allen, P. A. Bandettini, V. D. Calhoun, M. Corbetta, S. Della Penna, J. H. Duyn, G. H. Glover, J. Gonzalez-Castillo, D. A. Handwerker, S. Keilholz, V. Kiviniemi, D. A. Leopold, F. de Pasquale, O. Sporns, M. Walter, and C. Chang. 2013. 'Dynamic functional connectivity: Promise, issues, and interpretations', Neurolmage, 80: 360-78.

Leung, R.C., A.X. Ye, S.M. Wong, M.J. Taylor, and S.M. Doesburg. 2014. 'Reduced beta connectivity during emotional face processing in adolescents with autism', Molecular Autism, 5: 51.

Liuzzi, L., L.E. Gascoyne, P. Tewarie, E.L. Barratt, E. Boto, and M. J. Brookes. 2016. 'Optimising experimental design for MEG resting state functional connectivity measurement', Neurolmage, In submission.

Marek, S., K. Hwang, W. Foran, M.N. Hallquist, and B. Luna. 2015. 'The Contribution of Network Organization and Integration to the Development of Cognitive Control.', PLOS Biol, 13: e1002328. doi: 10.1371/journal.pbio.28. eCollection 2015 Dec.

Muthukumaraswamy, S.D. 2013. 'High-frequency brain activity and muscle artifacts in MEG/EEG: a review and recommendations', Frontiers in Human Neuroscience, 7: 138.

Nolte, G., O. Bai, L. Wheaton, Z. Mari, S. Vorbach, and M. Hallett. 2004. 'Identifying true brain interaction from EEG data using the imaginary part of coherency', Clinical Neurophysiology, 115: 2292 - 307.

O'Neill, G. C., E. L. Barratt, B. A. E. Hunt, P. K. Tewarie, and M. J. Brookes. 2015. 'Measuring electrophysiological connectivity by power envelope correlation: A technical review on MEG methods', Physics in Medicine and Biology, 60: R271-R95.

O'Neill, G. C., M. Bauer, M. W. Woolrich, P. G. Morris, G. R. Barnes, and M. J. Brookes. 2015. 'Dynamic recruitment of resting state sub-networks', Neurolmage, 115: 85-95.

O’Neill, G.C., P.K. Tewarie, D. Vidaurre, M.W. Woolrich, and M.J. Brookes. 2017. 'Dynamics of large-scale electrophysiological networks', Neurolmage, In submission.

Rezek, I., and S. Roberts. 2005. 'Ensemble hidden markov models with extended observation densities for biosignal analysis.' in Dybowski R Husmeier D, Roberts S (ed.), Probabilistic Modeling in Bioinformatics and Medical Informatics. (London: Springer London).

Robinson, S., and J. Vrba. 1998. 'Functional Neuroimaging by synthetic Aperture Magnetometry.', In Recent Advances in Biomagnetism, ed. Yoshimoto T, Kotani M, Kuriki S, Karibe H, Nakasato N, Tohoku Univ. Press, Sendai, Japan.: 302-05.

Sarvas, J. 1987. 'Basic mathematical and electromagnetic concepts of the biomagnetic inverse problem', Physics in Medicine and Biology, 32: 11 - 22.

Schäfer, C.B., B.R. Morgan, A.X. Ye, M.J. Taylor, and S.M. Doesburg. 2014. 'Oscillations, networks and their development: MEG connectivity changes with age', Human Brain Mapping, 35: 5249-61.

Sekihara, K., S.S. Nagarajan, D. Poeppel, and A. Marantz. 2004. 'Performance of an MEG adaptivebeamformer source reconstruction technique in the presence of additive low-rank interference', IEEE Trans. Biomed. Eng., 51: 90-99.

Stam, C.J., G. Nolte, and A. Daffertshofer. 2007. 'Phase Lag Index: Assessment of Functional Connectivity From Multi Channel EEG and MEG With Diminished Bias From Common Sources', Human Brain Mapping, 28: 1178 - 93.

Supekar, K. et al. 2013. 'Brain hyperconnectivity in children with autism and its links to social deficits', Cell Rep, 5: 738-47.

Tewarie, P., M. G. Bright, A. Hillebrand, S. E. Robson, L. E. Gascoyne, P. G. Morris, J. Meier, P. Van Mieghem, and M. J. Brookes. 2016. 'Predicting haemodynamic networks using electrophysiology: The role of non-linear and cross-frequency interactions', Neurolmage, 130: 273-92.

Tewarie P.K., A. Hillebrand, E. van Dellen, M.M. Schoonheim, Barkhof F., C.H. Polman, C. Beaulieu, G. Gong, B.W. van Dijk, and C.J. Stam. 2014. 'Structural degree predicts functional network connectivity: A multimodal resting-state fMRI and MEG study', Neurolmage, 97: 296-307. 
Tzourio-Mazoyer, N., B. Landeau, D. Papathanassiou, F. Crivello, O. Etard, N. Delcroix, and B. Mazoyer. 2002. 'Automated Anatomical Labeling of activations in SPM using a Macroscopic Anatomical Parcellation of the MNI MRI single-subject brain', Neurolmage, 15 273-89.

Vidaurre, D., A. J. Quinn, A. P. Baker, D. Dupret, A. Tejero-Cantero, and M. W. Woolrich. 2016. 'Spectrally resolved fast transient brain states in electrophysiological data', Neurolmage, 126: 81-95.

Wens, V., B. Marty, A. Mary, M. Bourguignon, M. Op de Beeck, S. Goldman, P. Van Bogaert, P. Peigneux, and X. De Tiege. 2015. 'A geometric correction scheme for spatial leakage effects in MEG/EEG seed-based functional connectivity mapping', Hum Brain Mapp, 36: 4604-21.

Whitham, E.M., K.J. Pope, S.P. Fitzgibbon, T. Lewis, C.R. Clark, S. Loveless, M. Broberg, A. Wallace, D. DeLosAngeles, P. Lillie, A. Hardy, R. Fronsko, A. Pulbrook, and J.O. Willoughby. 2007. 'Scalp electrical recording during paralysis: quantitative evidence that EEG frequencies above $20 \mathrm{~Hz}$ are contaminated by EMG.', Clin. Neurophysiol., 118: 1877-88.

Woolrich, M.W., A. Baker, H. Luckhoo, H. Mohseni, G. Barnes, M.J. Brookes, and L. Rezek. 2013. 'Dynamic state allocation for MEG source reconstruction', Neurolmage, 77: 77-92.

Ye, A.X., R.C. Leung, C.B. Schäfer, M.J. Taylor, and S.M. Doesburg. 2014. 'Atypical resting coherence in autism spectrum disorder', Human Brain Mapping, 35: 6049-66.

You, X. et al. 2013. 'Atypical modulation of distant functional connectivity by cognitive state in children with Autism Spectrum Disorders', Frontiers in Human Neuroscience, 7: 482.

Zimmermann, R., and E. Scharein. 2004 'MEG and EEG show different sensitivity to myogenic artifacts.', Neurol Clin Neurophysiol., 78. 